\title{
El ser humano como ser ocupacional
}

\author{
Sergio Santos del Riego
}

Catedrático EU. de Medicina Física y Rehabilitación. Vicedecano de la Facultad de Ciencias de la Salud, Universidad de A Coruña. Presidente de la Conferencia Nacional de Directores de Escuelas Universitarias de Terapia Ocupacional (CNDEUTO)

\section{Terapia Ocupacional: aproximación conceptual}

La Terapia Ocupacional (TO) es una profesión enmarcada en las ciencias de la salud y la sociedad que utiliza como medio terapéutico la actividad significativa o propositiva (ocupación) dirigida y programada con un fin preventivo o terapéutico, para facilitar la adaptación ecológica y funcional de cada persona, sea considerada como un paciente, sea considerada como un usuario/cliente; y conforma una doctrina específica centrada en la Ciencia Ocupacional. Sharrot y Yerxa $^{1}$ han argumentado a favor de seguir utilizando el término paciente para describir a las personas que utilizan los servicios del terapeuta ocupacional, ya que el término contiene implicaciones éticas y morales. Sin embargo, la filosofía general de actuación, salvo en los modelos y marcos de referencia médicos y de rehabilitación, prefiere usar el término usuario o cliente ${ }^{1}$. Según Gary Kielhofner ${ }^{2}$, la ocupación es la actividad principal del ser humano en todas sus vertientes, fruto de un proceso evolutivo que culmina en el desarrollo de sus necesidades biológicas, psicológicas y sociales. La TO estudia la ocupación de las personas en las áreas de autocuidado/automantenimiento, productividad y ocio, tiempo libre y juego ${ }^{3,4}$.

Las definiciones enunciadas de TO desde los diferentes organismos nacionales e internacionales son múltiples y tienen un eje común centrado en la ocupación con potencialidad terapéutica curativa, algunas se exponen en las tablas 1 y 2.

La TO se sustenta conceptualmente en tres pilares imprescindibles: el paciente/usuario, el terapeuta ocupacional y la ocupación. Nunca se hará TO si falta uno de estos tres pilares. Una buena empatía e interacción es la base de la relación del paciente/usuario con el terapeuta ocupacional. Además, el paciente/usuario y la ocupació mantienen una relación que define el grado de dependencia del individuo en el entorno 5 .

\section{Campos y ámbitos de actuación}

Una persona es subsidiaria de tratamiento por el terapeuta ocupacional cuando presenta alteraciones en el desempeño de su ocupación, bien por causas resultado de una lesión o enfermedad que altera las funciones motoras, sensoriales, cognitivas y/o psicológicas o bien por causas derivadas del entorno físico, social y cultural ${ }^{6}$.

Los terapeutas ocupacionales pueden desarrollar su desempeño profesional en diversos campos que se detallan en la tabla 3. Los ámbitos de actuación para los profesionales de TO son muy variados. Así, estos profesionales desarrollan su trabajo en centros de diversos ámbitos, que se exponen en la tabla 4, tanto de carácter público como privado ${ }^{7}$. 
TABLA 1. Definiciones de Terapia Ocupacional nacionales

Asociación Profesional Española de Terapeutas Ocupacionales (APETO)
"Una profesión sociosanitaria, que a través de la valoración de las capacidades y problemas físicos, psicológicos y sociales del individuo pretende, con un adecuado tratamiento, capacitarle para alcanzar el mayor grado de independencia posible en su vida diaria, contribuyendo a la recuperación de su enfermedad y/o facilitando la adaptación a su discapacidad"

"Una disciplina sociosanitaria que evalúa la capacidad de la persona para desempeñar las actividades de la vida cotidiana e interviene cuando dicha capacidad corre un riesgo o está dañada por cualquier causa"

TABLA 2. Definiciones de Terapia Ocupacional internacionales

Federación Mundial de Terapia Ocupacional (WFOT)

Asociación Americana de Terapia Ocupacional (AOTA)

Comité de Terapia Ocupacional para la Comunidad Europea (COTEC)
"Una profesión de la salud basada en la utilización de la actividad propositiva para promover la salud y el bienestar en todos los aspectos de la vida diaria"

"El tratamiento de las condiciones físicas y psiquiátricas mediante actividades específicas para ayudar a la gente a buscar su máximo nivel de función e independencia en todos los aspectos de su vida cotidiana"

"El uso de la actividad propositiva o de intervenciones encaminadas a promover la salud y conseguir resultados funcionales en el sentido de independencia personal"

"El análisis y aplicación de ocupaciones seleccionadas, de la vida diaria, que tienen un significado y propósito para el individuo y le capacita para desarrollar, recuperar, fortalecer o prevenir la pérdida de hábitos, habilidades, tareas, rutinas o roles ocupacionales que ha realizado en el pasado o está aprendiendo a realizar para participar en la medida de lo posible como miembro de su entorno personal, social, cultural y económico"

"La ciencia que valora y trata a la gente usando actividades propositivas para prevenir la discapacidad y desarrollar la independencia funcional

TABLA 3. Campos de intervención en Terapia Ocupacional

Según modelos localistas

Según modelos poblacionales

Según modelos sensoriales

Según modelos de rehabilitación laboral

Según modelos de reinserción social, familiar y educativa

Según modelos fundamentados en las nuevas tecnologías informáticas y de apoyo a la rehabilitación

Otros
Rehabilitación Osteo-Muscular

Rehabilitación Neurológica y Neuro-Traumatológica

Rehabilitación en Salud Mental

Pediatría

Geriatría/Gerontología

Eje de Integración Sómato-Sensorial (tacto, propiocepción y cinestesia)

Rehabilitación de Personas Ciegas o Ambliopes (básica y visual)

Rehabilitación de Personas con Sordera

Discapacidad y marginación social

Discapacidad y marginación social (alcoholismo, drogodependencias, maltrato en mujeres, ancianos o niños, prostitución, pobreza, sin techo, entre otros)

Equipamientos adaptados 
TABLA 4. Ámbitos en Terapia Ocupacional ${ }^{7}$

\begin{tabular}{|c|c|}
\hline Sanitario & $\begin{array}{l}\text { Hospitales Generales Públicos } \\
\text { Hospitales Universitarios } \\
\text { Centros Hospitalarios Monográficos } \\
\text { Hospitales de Día } \\
\text { Ambulatorios de Especialidades } \\
\text { Centros de Atención Primaria } \\
\text { Atención Domiciliaria } \\
\text { Hospitales y Clínicas Privadas } \\
\text { Residencias de Ancianos } \\
\text { Centros Gerontológicos de Estancias Diurnas } \\
\text { Mutuas de Accidentes } \\
\text { Balnearios }\end{array}$ \\
\hline Sociosanitario y Social & $\begin{array}{l}\text { Ayuntamientos/Servicios Sociales } \\
\text { Atención a Personas con Drogadicción } \\
\text { Centros de Día } \\
\text { Centros Especiales de Empleo/Ocupacionales } \\
\text { Centros de Reinserción de Minusválidos Físicos } \\
\text { Organizaciones no Gubernamentales (ONG) } \\
\text { Fundaciones y Asociaciones } \\
\text { Instituciones Penitenciarias }\end{array}$ \\
\hline Educativo & $\begin{array}{l}\text { Centros de Enseñanza } \\
\text { Guarderías Infantiles } \\
\text { Colegios de Integración y Educación Especial } \\
\text { Asociaciones } \\
\text { Bibliotecas }\end{array}$ \\
\hline $\begin{array}{l}\text { Docencia } \\
\text { Asesoramiento y otros }\end{array}$ & $\begin{array}{l}\text { Innovación, Investigación y Desarrollo } \\
\text { Casas de Ortopedia } \\
\text { Prevención de Riesgos Laborales } \\
\text { Libre Ejercicio }\end{array}$ \\
\hline
\end{tabular}

\section{Objetivos y marcos de referencia}

El terapeuta ocupacional es un profesional que participa, formando parte de diversos equipos de carácter multidisciplinar e interdisciplinar, en la prevención de la enfermedad y de la discapacidad. Así mismo, promueve el desempeño ocupacional del paciente/usuario a través de la evaluación, modulación y aplicación de actividades seleccionadas de acuerdo con las dificultades, necesidades, potencialidad, historia de vida, condición sociocultural y proyectos futuros de la persona con discapacidad o marginada socialmente ${ }^{5}$.

El objetivo específico del médico rehabilitador en los Servicios de Medicina Física y Rehabilitación está orientado a detectar y definir las necesidades ocupacionales del paciente, para que el terapeuta ocupacional pueda actuar, a posteriori, con las intervenciones ocupacionales terapéuticas adecuadas, tal y como se define en el Estatuto del Personal Sanitario no Facultativo de las Instituciones de la Seguridad Social, aprobada por Orden de 26 de diciembre de 1986 del Ministerio de Sanidad y Consumo ${ }^{8,9}$.

Los marcos de referencia teóricos aplicados en la rehabilitación de personas con discapacidad son de diversa índole y cada uno de ellos define principios y fronteras orientados en función de la etiología de la discapacidad (o de la marginación social). Gómez Tolón ${ }^{10,11}$, médico rehabilitador, define tres marcos de enfoque organicista, tres de enfoque mentalista y otros tres mixtos. Y Polonio ${ }^{12}$, terapeuta ocupacional, expone una clasificación con marcos teóricos de referencia fisiológicos o psicológicos. Esta divergencia en la definición del número y tipo de marcos de referencia demostrada entre los autores españoles tiene una clara correspondencia en la cantidad y calidad de marcos referenciados por diferentes autores extranjeros ${ }^{13}$.

Lo habitual es que un profesional de TO actúe con uno o dos marcos teóricos de referencia, ya que el conocimiento y la aplicación práctica de varios es prácticamente imposible. La elección del marco de referencia por el terapeuta ocupacional está determinada por las características del paciente/usuario y por la filosofía del centro de trabajo en el que desarrolla su profesión. Algunos de los marcos de referencia más significativos se aplican siguiendo diversos modelos, tales como los de ocupación humana de Gary 
Kielhofner, de desempeño ocupacional de la Asociación Americana de Terapia Ocupacional, de las habilidades adaptativas de Mosey, de la práctica centrada en el cliente (modelo canadiense) y otros ${ }^{12,14}$.

\section{Evaluación en Terapia Ocupacional}

El proceso de evaluación va orientado a descubrir si el paciente enfoca adecuadamente las tareas y las expectativas propias de su rol y a determinar las perspectivas y necesidades ocupacionales en todas las áreas de desempeño ocupacional ${ }^{15,16}$. La evaluación se efectúa teniendo en consideración el desarrollo humano y abarca aspectos físicos, psíquicos, sensoriales y sociales. Se deben analizar los requerimientos laborales y del tiempo libre del paciente (niño en edad escolar, ama de casa, actividades productivas de cada adulto, anciano). Según Laver Fawcett ${ }^{13}$, la evaluación se puede hacer "de abajo arriba", es decir, comenzar analizando los componentes de la disfunción ocupacional específica y, también, de "arriba abajo", sometiendo a un intenso estudio el rol del paciente/usuario en todas las áreas de desempeño ocupacional. El primero de los métodos es el usado con más frecuencia en las unidades de TO de los Servicios de Medicina Física y Rehabilitación y de Psiquiatría. Habitualmente, en la mayoría de los centros hospitalarios españoles, los pacientes son derivados al terapeuta ocupacional desde el médico rehabilitador o psiquiatra, comunicándose ambos profesionales de la salud mediante un método indirecto: la ficha médica de prescripción del tratamiento. Esta ficha informa de los apellidos y el nombre del paciente, la edad, la profesión, el estado civil, el domicilio, el modo de contacto y el diagnóstico clínico y de la discapacidad. Además, se realiza una aproximación general al diagnóstico ocupacional del paciente, en el que se definen las necesidades detectadas en todas las áreas de desempeño ocupacional, aspectos de la adaptación funcional, aspectos relacionados con ayudas técnicas, prótesis, ortesis o de otras funciones específicas del terapeuta ocupacional, así como las contraindicaciones y aspectos tanto de índole psicológica como de la historia laboral y social del paciente. En algunos Servicios de Medicina Física y Rehabilitación se está valorando la posibilidad de sustituir estas fichas de tratamiento por sesiones clínicas multidisciplinares e interdisciplinares periódicas y regulares, coordinadas por el médico responsable. El terapeuta ocupacional también obtiene datos de otras fuentes como son los informes del médico rehabilitador, de enfermería, de psicología y de otros profesionales de la salud implicados.

La información se complementa con la observación directa informal o estandarizada, las entrevistas aleatorias u organizadas con el paciente/usuario, la familia y otros profesionales de la salud y la realización de escalas de valoración, baterías y pruebas específicas, que permiten realizar un registro completo personalizado, denominado historia ocupacional.

En la fase inicial todas las actuaciones del profesional están orientadas a mejorar la empatía terapeutapaciente, a promover la autoconciencia en el individuo de las necesidades ocupacionales y al establecimiento de las prioridades y de los objetivos a corto, medio y largo plazo. A posteriori , se continuará con la selección y el diseño de la actividad ocupacional y la organización de un plan de tratamiento personalizado en un marco teórico de referencia determinado ${ }^{14}$. La familia debe estar implicada en el proceso terapéutico del paciente.

Es importante conocer los planteamientos terapéuticos concomitantes, a efectos de facilitar la coordinación en el tiempo y en el espacio. En los Servicios de Medicina Física y Rehabilitación, el médico es el responsable de planificar las actuaciones de todos los profesionales implicados en el proceso clínico y terapéutico que afectan al paciente con discapacidad.

Con posterioridad, se efectúan evaluaciones ocupacionales provisionales de la evolución del paciente mientras se realiza el tratamiento. Una vez finalizada la intervención, se procede a la realización de una valoración ocupacional final que detecta el éxito del tratamiento, medido por los objetivos conseguidos, y el fracaso, por aquellos otros no alcanzados ${ }^{14}$.

El terapeuta debe redactar la historia ocupacional como una fuente documental que estará formada por la suma del informe de evaluación inicial (capacidades funcionales y necesidades ocupacionales iniciales), las baterías realizadas, el informe con el programa de tratamiento, los sucesivos informes provisionales de evolución y la valoración-evaluación final con un resumen de los resultados/capacidades definitivos. 


\section{Consideraciones finales}

1. La TO se modula en una relación de empatía entre el terapeuta ocupacional y el paciente, mediante el análisis y el uso de la actividad ocupacional con significado (ocupación), la adaptación funcional del/al entorno y la rehabilitación terapéutica de personas con discapacidad (o marginadas socialmente) que pretenden normalizar al máximo su vida.

2. La TO española está participando en un proceso evolutivo muy dinámico que se traduce en un mayor impulso en todos los ámbitos, heredero de la tradición histórica y del desarrollo de esta profesión en la mayoría de países occidentales ${ }^{17,18}$.

3. En la actualidad, la mayoría de profesionales de TO están desempeñando su labor asistencial en las Residencias y Centros de Día de ancianos, centros en los que no es habitual la presencia del especialista en Medicina Física y Rehabilitación ${ }^{18}$. Este contexto sugiere que debe potenciarse la presencia del médico rehabilitador en éstos y otros dispositivos extrahospitalarios.

4. En España, fruto de la expansión extrahospitalaria de la TO realizada en esta última década, se encuentra que la figura del terapeuta ocupacional está en una menor proporción en los ámbitos de salud mental y de rehabilitación física ${ }^{18}$.

5. Estos profesionales emplean marcos teóricos de referencia y diversos modelos, algunos de ellos muy novedosos, que se han ido desarrollado en los dos últimos siglos de historia de la TO.

6. El fundamento de la TO se basa en un modelo de uso preventivo y terapéutico de la Ciencia Ocupacional para la función y la calidad de la vida del paciente/usuario.

\section{BIBLIOGRAFÍA}

1. Bauer D. Rehabilitación: enfoque integral. Barcelona: Masson-Salvat; 1992.

2. Kielhofner G. Conceptual foundations of occupational therapy. Philadelphia: FA Davis; 1992.

3. Hooper B, Wood W. Pragmatism and structuralism in occupational therapy: the long conversation. Am J Occup Ther. 2002;56:40-50.

4. Cruz Hernández J. La clasificación internacional del funcionamiento, de la discapacidad y de la salud (CIF) y la medicina de Rehabilitación. Rehabilitación (Madr). 2002;36:1-2.

5. Galvez B, Isaac MV, Muñoz C, Riveros ME, Correa I, Valdés A. La atención primaria en salud y su relación con la Terapia Ocupacional. Nuevos métodos de educación sanitaria en la atención primaria de salud. Organización Mundial de la Salud (OMS): Serie Informes Técnicos; 1982.

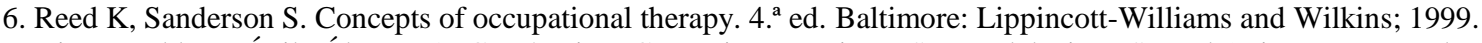

7. Viana Moldes I, Ávila Álvarez A, García Pinto C, Pereira Loureiro J, Santos del Riego S, et al (primera y segunda promoción de alumnado de Terapia Ocupacional). Díptico de promoción de la Terapia Ocupacional 2001-2002. A Coruña: Facultad de Ciencias de la Salud-Universidad de A Coruña; 2002

8. Orden Ministerial de 26 de diciembre de 1986 del Ministerio de Sanidad y Consumo, por la que se aprueba el Estatuto del Personal Sanitario no Facultativo de las Instituciones de la Seguridad Social.

9. Santos del Riego S, Hernández Herrero D. Planificación del alta en centros de rehabilitación aguda y subaguda. En: Miangolarra Page JC, Alguacil Diego AM, Águila Maturana A, editores. Rehabilitación: Clínica integral. Funcionamiento y discapacidad. Barcelona: Masson; 2003. p. 122-8.

10. Gómez Tolón J. Fundamentos metodológicos de la Terapia Ocupacional. Zaragoza: Mira Editores; 1997.

11. Gómez Tolón J. Habilidades y destrezas en Terapia Ocupacional. Zaragoza: Mira Editores; 2000.

12. Polonio López B, Durante Molina P, Noya Arnaiz B. Conceptos fundamentales de Terapia Ocupacional. Madrid: Médica Panamericana; 2001.

13. Turner A, Foster M, Johnson SE. Terapia ocupacional y disfunción física. Principios, técnicas y práctica. $5 .^{a}$ ed. Madrid: Elsevier España; 2003.

14. Romero Ayuso D, Moruno Miralles P. Terapia ocupacional. Teoría y técnicas. Barcelona: Masson; 2003.

15. Hagedorn R. Foundations for practice in occupational therapy. 2. ${ }^{a}$ ed. Edimburgo: Churchill Livingstone; 1997.

16. Hagedorn R. Tools for practice in occupational therapy. A structured approach to core skills and processes. Edimburgo: Churchill Livingstone; 2000.

17. Libro Blanco de la Terapia Ocupacional de la Conferencia Nacional de Directores de Escuelas Universitarias de Terapia Ocupacional. Zaragoza: Servicio de Publicaciones de la Universidad de Zaragoza; 2004.

18. Graduado de Terapia Ocupacional. Conferencia Nacional de Directores de Escuelas Universitarias de Terapia Ocupacional. Página web de la Facultad de Ciencias de la Salud de la Universidad de A Coruña [en línea] 2004 [fecha de acceso 4 de Octubre de 2004]; Descarga de archivos (documentos): 120. URL disponible en: http://www.fcs.udc.es 